lacunar infarction, presence of headache at stroke onset decreased early neurological recovery from $26.2 \%$ to $19.2 \%$. Absence of limitation at discharge was less frequent in subjects with supratentorial white matter lesions than in those with other types of infarction (15.1\% vs $25.1 \%$ ); this result was duplicated in the subgroup of patients without headache. In multivariate analysis, both absence of headache in deep brain gray matter or brainstem and presence of dysarthria-clumsy hand syndrome predicted good outcome.

These results, say the authors, point to an important role for glutamate in lacunar infarction and cerebral ischemia; synaptic glutamate receptors are mostly located in the deep brain gray matter or brainstem, and headache in patients with lacunar infarction could be due to biomechanical (inflammatory or neuroexcitotoxic) rather than mechanical aspects. Glutamate has additionally been proposed to exert a neurotoxic effect in deep brain regions but not white matter ischemic lesions. Further research into the excitotoxic activity of glutamate in patients with lacunar infarction could therefore be beneficial.

Original article Arboix A et al. (2006) Clinical implications of headache in lacunar stroke: relevance of site of infarct. Headache 46: 1172-1180

\section{Differences in the pathogenesis of migraine and cluster headache}

Abnormalities in the trigemino-cervical reflex have been associated with headache. To assess the role of the trigeminal system in head pain, researchers in Italy studied the response in the sternocleidomastoid muscle to stimulation of the infraorbital nerve in 30 patients with migraine, 10 patients with cluster headache and 15 healthy controls.

Compared with controls, the trigeminocervical response was abnormal during headache in 25 patients with migraine and 5 patients with cluster headache. In the inactive phase, an abnormal response was found in 20 patients with migraine and 1 patient with cluster headache. The observed abnormalities were unilateral in cluster headache and bilateral in migraine. Differences in responses were significant $(P<0.001)$ between all patients with headache and controls during the headache phase. In the inactive phase, differences between migraine patients and controls were significant $(P<0.001)$, but there was no significant difference between controls and patients with cluster headache.

The authors state that the bilateral nature of the abnormal response and the persistence of the abnormality in the inactive phase indicate a centrally located brainstem dysfunction in patients with migraine and suggest the involvement of central pain mechanisms in the disease. The results of the study offer support for the theory that abnormal brainstem modulation of nociceptive afferent neurons is involved in migraine pathogenesis. The authors say that different mechanisms are likely to be responsible for cluster headache, however.

Original article Nardone R et al. (2006) Trigemino-cervical reflex abnormalities in patients with migraine and cluster headache. Headache [doi: 10.1111/j.1526-4610.2006.00529.x]

\section{Increase in seizure frequency associated with common hormone replacement therapy}

Results of a recent double-blind, placebocontrolled trial indicate that hormone replacement therapy (HRT) can increase seizure frequency in patients with epilepsy.

Harden and colleagues recruited postmenopausal women with partial epilepsy (mean age 53 years) who were within 10 years of their last menses and were taking stable doses of antiepileptic drugs. After a 3-month prospective baseline period, 21 patients were randomized to receive daily placebo $(n=6)$, a daily single dose of conjugated equine estrogens plus medroxyprogesterone acetate (CEE/MPA; $n=8)$, or a daily double dose of CEE/MPA ( $n=7)$. Patients were using an average of one antiepileptic drug (range $0-3)$, and all met the standard medical criteria for the use of HRT prior to July 2002.

Five out of seven subjects taking double doses of CEE/MPA experienced increased seizure frequency of at least one seizure type, compared with four out of eight subjects taking single doses of CEE/MPA and one out of six subjects taking placebo. Two patients who were taking lamotrigine and CEE/MPA experienced a reduction in their lamotrigine levels.

The authors conclude that CEE/MPA might not be the best HRT regimen for women with partial epilepsy, although they note that their 